Instituto Internacional de Investigación y Desarrollo Tecnológico Educativo INDTEC, C.A.

DOI: https://doi.org/10.29394/Scientific.issn.2542-2987.2020.5.16.11.208-229

OAI-PMH: http://www.indteca.com/ojs/index.php/Revista Scientific/oai

Artículo Original / Original Article

\title{
Comportamientos generacionales y contenidos informativos que construyen el mundo de las Fake News en Ecuador
}

Autoras: Michelle Alexandra Benítez Vite Universidad Internacional del Ecuador, UIDE mia77bv@gmail.com Quito, Ecuador https://orcid.org/0000-0002-8567-6370

Patricia Elizabeth Hidalgo Albuja Universidad Internacional del Ecuador, UIDE phidalgo@uide.edu.ec Quito, Ecuador https://orcid.org/0000-0002-0735-8991

\section{Resumen}

El presente trabajo se basa en una revisión bibliográfica que describe cómo funcionan las noticias falsas, su creación, objetivos y el entorno en el que se desarrollan. Partiendo de ello, el objetivo de estudio es analizar que comportamientos y tipos de contenido configuran el mundo de las noticias falsas en el Ecuador. Tomando elementos de teorías como la del Modelo de la probabilidad de elaboración de la persuasión (ELM), el modelo de comunicación de Costa (2012) y las diferencias generacionales planteadas por Kotler y Keller (2012), se crea un modelo de investigación conceptual y se extraen posibles relaciones que arrojan preguntas de investigación sobre el tema. A través de encuestas y análisis de casos, se llega a la conclusión de que ciertos comportamientos ante las noticias falsas tienen relación directa con la edad, como preferencias de medios informativos y carácter de contenidos favoritos. Sin embargo, algunas de las relaciones planteadas arrojan resultados inconcluyentes que deberían ser explorados en futuras investigaciones.

Palabras clave: política; redes sociales; información.

Cómo citar este artículo:

Benítez, M., \& Hidalgo, P. (2020). Comportamientos generacionales y contenidos informativos que construyen el mundo de las Fake News en Ecuador. Revista Scientific, 5(16), 208-229, e-ISSN: 25422987. Recuperado de: https://doi.org/10.29394/Scientific.issn.2542-2987.2020.5.16.11.208-229

Fecha de Recepción: 13-12-2019
Fecha de Aceptación: 25-03-2020
Fecha de Publicación:

05-05-2020 
OAI-PMH: http://www.indteca.com/ojs/index.php/Revista_Scientific/oai

Artículo Original / Original Article

\title{
Generational behaviors and informative content that build the world of Fake News in Ecuador
}

\begin{abstract}
This work is based on a bibliographic review that describes how fake news works, its creation, objectives and the environment in which they develop. Based on this, the objective of the study is to analyze what behaviors and types of content shape the world of fake news in Ecuador. Taking elements of theories such as the model of the probability of elaboration of persuasion (ELM), the communication model of Costa (2012) and the generational differences raised by Kotler and Keller (2012), a conceptual research model is created and possible relationships are extracted that They throw research questions on the subject. Through surveys and case analysis, it is concluded that certain behaviors in the face of false news are directly related to age, such as media preferences and favorite content character. However, some of the relationships raised show inconclusive results that should be explored in future research.
\end{abstract}

Keywords: politics; social media; information.

How to cite this article:

Benítez, M., \& Hidalgo, P. (2020). Generational behaviors and informative content that build the world of Fake News in Ecuador. Revista Scientific, 5(16), 208-229, e-ISSN: 2542-2987. Recovered from: https://doi.org/10.29394/Scientific.issn.2542-2987.2020.5.16.11.208-229

Date Received: 13-12-2019
Date Acceptance:

25-03-2020
Date Publication:

05-05-2020 


\section{Introducción}

\subsection{Noticias falsas: definición}

Las noticias falsas, que para Gelfert (2018): son "[la] presentación deliberada de afirmaciones (típicamente) falsas o engañosas [como si fueran noticias]" (pág. 108); han adquirido relevancia en varios campos teóricos y prácticos en la última década. Se han realizado documentales, estudios y críticas constantes sobre el nivel de inserción que este tipo de contenido tiene en el mundo digital. Según Allcott, Gentzkow y Yu (2019): un monitoreo realizado en Estados Unidos a 570 sitos de noticias falsas, reveló que estos producen al menos 70 millones de interacciones al mes en Facebook con sus contenidos. Esto, viene a ser casi la mitad de las interacciones que los sitios grandes como CNN, New York Times y Fox News consiguen en el mismo periodo de tiempo.

En Ecuador, el fenómeno de las noticias falsas se produce especialmente en las redes sociales donde se aprovechan contextos electorales o políticos para la creación de contenido que tiene como fin crear oposición hacia figuras o temas específicos. Las noticias falsas de manera general, son un resultado de los cambios tecnológicos y los comportamientos que usuarios han desarrollado ante los mismos. Ello acarrea una serie de modificaciones y planteamientos, tanto éticos como económicos, que recaen sobre el creador y el receptor de contenidos mediáticos. Las noticias falsas han aprovechado la versatilidad de formatos para mezclarse en un mar de información en el que los usuarios de redes sociales navegan cada día sin saber que es verdad y que no.

La verdad se ha convertido en posverdad y en la época posmoderna, dice Vives (2019a): se parte de la "premisa de que nada es verdad y todo es posible" (pág. 290); para dudar de todo e interpretarlo como nuestra percepción dicta, no como es. Esto se deriva de la idea de que, en una sociedad pluralista como la nuestra, según Vives (2019b): "resulta lógico tratar 
de destacar la importancia de múltiples voces y contribuir a la negación del poder de una sola voz" (pág. 294). Múltiples voces se prestan a múltiples perspectivas de un evento que muchas veces pueden exagerar, omitir, modificar o tergiversar los hechos para luego publicarlos como noticias.

\subsection{El entorno en el que se desarrollan las noticias falsas}

Tener un panorama completo del escenario en el que las noticias falsas se desarrollan implica un análisis de usuarios, contenido y objetivos derivados de la producción de noticias falsas.

Un enfoque teórico interesante hace referencia a la ecología de los medios digitales. Señalando a Bakir y McStay (2017): las noticias falsas son una consecuencia lógica de las cinco características de la ecología de los medios digitales que son: 1). el declive financiero de las noticias en formatos tradicionales; 2). la inmediatez creciente del proceso de generación de noticias; 3). la rápida circulación de la desinformación por medio de contenidos creados por usuarios de redes; 4). la naturaleza emocional de los discursos en línea; y 5). el creciente número de individuos que generan ingresos económicos gracias a los algoritmos de las redes sociales. Estos postulados tratan de describir el entorno digital actual.

Un segundo enfoque, que parte de ideas similares, hace hincapié en la industria SEO que precede a las redes sociales. Señala Mustafaraj y Metaxas (2017a): que una versión menos sofisticada y más general de lo que podrían ser consideradas hoy noticias falsas, antes se hablaba de "propaganda online" (pág. 3). Paralelamente, Mustafaraj y Metaxas (2017b): mencionan que los contenidos políticos y religiosos cargados de sesgos, han sido promovidos y posicionados en la web gracias al Search Engine Optimization durante años. Esto llevó a la aparición de un segundo fenómeno los "clickbaits" o "ciberanzuelos", que vienen a ser un importante componente de las noticias falsas. Estos se pueden definir como titulares que llaman la atención con fin 
de mantener al usuario en una página o sitio web el mayor tiempo posible. Los tiempos largos de visualización son atractivos para los anunciantes que pagarán por tener un espacio publicitario en sitios con contenidos que atraen audiencias grandes.

Es importante resaltar que muchos de los estudios sobre noticias falsas en Ecuador, hacen referencia a contextos políticos donde se usan y no sobre mecanismos de funcionamiento. Pero los postulados sobre la ecología de los medios digitales y la industria SEO son aplicables al entorno digital ecuatoriano por el carácter global de la nueva economía que, de acuerdo con Calderón (2019): se basa en el procesamiento de la información.

\subsection{Porque se comparten las noticias falsas}

En cuanto a la circulación de noticias falsas, un estudio publicado en el año 2019 por la Journal of Retailing and Consumer Services, llegó a varias conclusiones que exploran la relación entre la importancia que le da el usuario a su reputación en línea y el contenido que comparte. Una de ellas es que los usuarios de las redes sociales que confían en la fuente de información tienen altas probabilidades de compartirlos con otros sin verificar su autenticidad antes. Pero, por otro lado, aquellos que tienden a volverse auto divulgadores de información y lo hacen con el fin de construir relaciones, se asegurarán de que la noticia sea verdadera para compartirla.

Sin embargo, cuando hablamos de individuos, también hay un grupo cuyas motivaciones se inclinan a la mera participación en redes, sin importar el contenido. Al respecto, Talwar, Dhir, Kaur, Zafar y Alrasheedy (2019): mencionan dos términos: El Fear of missing out (FoMo) o miedo a perderse de algo, que lleva a los usuarios a compartir noticias falsas porque, no compartir algo popular, eleva los niveles de ansiedad de los individuos; y el segundo término, el social media fatigue (SMF), es definido por Ravindran, Yeow y Hoe (2014): como "una experiencia subjetiva y multidimensional que abarca 
sentimientos como cansancio, irritación, enojo, decepción, pérdida de interés o reducción de la necesidad/motivación asociada con varios aspectos del uso de redes sociales" (pág. 2317). El SMF incita a los usuarios perezosos a compartir noticias (falsas o no) como una manera de mantenerse activos en redes sin esforzarse demasiado.

\subsection{Teorías de apoyo}

Como ya se estableció, el fin de las noticias falsas es el persuadir, ya sea en acciones o en opiniones a una audiencia específica. Para el presente estudio se consideró el modelo Elaboration Likelihood Model of Persuasion (ELM) o Modelo de la probabilidad de elaboración de la persuasión que trata de explicar el fenómeno de la persuasión de contenidos. Elaboration en inglés hace referencia a la acción de desarrollar o reflexionar sobre un contenido. Este modelo, propuesto en los 80's por Petty y Cacciopo (1986): dice que la comunicación persuasiva logra modificar actitudes de las audiencias a través de dos posibles caminos: una ruta central y una periférica. La ruta central es aquella donde el usuario considera los argumentos del mensaje en base a los parámetros que contenidos previos han establecido en su mente. La ruta central implica un análisis más profundo del contenido, comparaciones, reflexiones, etc.

Si la audiencia está motivada a elaborar sobre un contenido y además tiene el conocimiento necesario para hacerlo, es más probable que cuestione aquello que se le presenta. Este tipo de persuasión es más efectivo porque el convencimiento viene del interior del individuo y va de acuerdo con sus valores propios.

El segundo tipo de persuasión surge de la ruta periférica, que considera señales externas al mensaje, y más relacionadas con el formato al momento de determinar el valor del contenido que está leyendo. En este caso, dice Maharjan (2018): que factores como atracción, autoridad y la llamada presión 
social toman fuerza. En este caso la audiencia se encuentra menos motivada a 'elaborar' sobre el contenido que se le presenta y por ende el análisis que hace sobre este es superficial. Tanto la persuasión generada por la ruta central como aquella generada por la ruta periférica, alcanzan diferentes niveles de convencimiento y, por ende, comportamientos más acordes al individuo y sus opiniones (ruta central) o a la presentación de los contenidos (ruta periférica).

El objetivo del presente estudio es analizar que comportamientos y tipos de contenido configuran el mundo de las noticias falsas en el Ecuador. Para analizar qué factores pueden influir en la credibilidad de las mismas, se usan elementos de la teoría del ELM y en base al material teórico presentado anteriormente se pretende responder a interrogantes relacionadas con usuarios y medios del país en un contexto político donde se presentaron noticias falsas.

\section{Metodología}

Para el desarrollo del presente estudio se propuso un modelo conceptual de investigación. Este tipo de modelos se basan en revisiones teóricas, que de acuerdo con Elangovan y Rajendran (2015a): sirven "para delinear los posibles cursos de acción o para presentar una idea o pensamiento" (pág. 1). De esta revisión se extraen conceptos que se relacionan entre sí para ser presentados en forma gráfica. Elangovan y Rajendran (2015b), además sostienen que:

Las definiciones básicas de una teoría, sus elementos y características (...) se entienden primero y se evalúan los diferentes procesos de construcción de la teoría. Los diversos conceptos se sintetizan y el proceso de desarrollo del modelo conceptual se explica sistemáticamente con una ilustración (pág. 43).

El modelo construido para esta investigación, tomó principios teóricos del proceso de comunicación, expuestos en la figura 1, rangos generacionales, 
presentados en la tabla 1, y componentes del Modelo de la probabilidad de elaboración ELM, plasmados en la figura 2, que fue explorado en la introducción. Se destacaron aquellos elementos que pueden tener una relación observable en el estudio.

Figura 1. Modelo de comunicación base, utilizado para la investigación, apoyado en el modelo de Costa (2012).

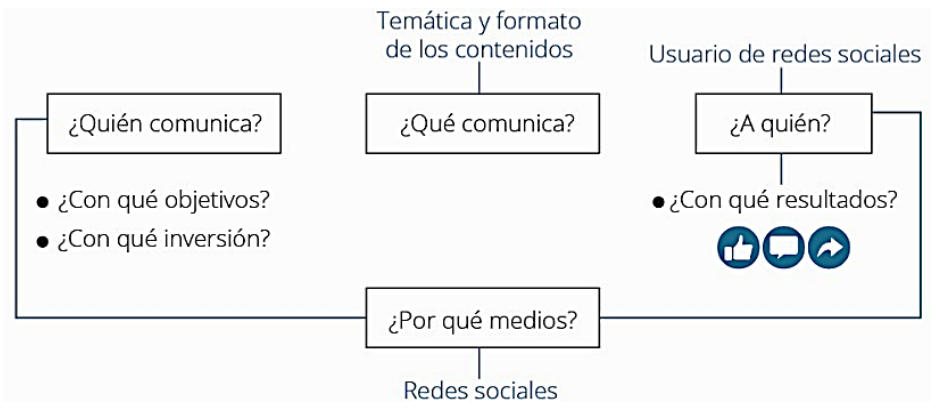

Fuente: Las Autoras (2019).

Al adaptar el modelo de comunicación propuesto por Costa (2012): a la temática de las fake news en redes sociales el que se comunica, a quién se comunica, porque medios y con qué resultados son preguntas que al ser investigadas proporcionan información clave sobre la interacción entre contenidos y usuarios.

Adicionalmente, se tomó la clasificación realizada por Kotler y Keller (2012): en su libro Dirección de Marketing, en combinación con aquella presentada en el Estudio global de la Compañía Nielsen (2015): sobre Estilos de Vida generacionales, para considerar rangos etarios como variable independiente. Los rangos etarios y sus nombres se resumen en la tabla 1.

Tabla 1. Generaciones por rango de edad, en base a revisión bibliográfica.

\begin{tabular}{|c|c|}
\hline Generación $Z$ & 0 a 24 años \\
\hline Generación $Y$ & 25 a 37 años \\
\hline Generación $X$ & 38 a 54 años \\
\hline Baby Boomers & 55 a 74 años
\end{tabular}

Fuente: Las Autoras (2019). 
OAI-PMH: http://www.indteca.com/ojs/index.php/Revista_Scientific/oai

\section{Artículo Original / Original Article}

Acorde con esto, el considerar el ELM, representado en la figura 2, con relación a cómo los contenidos informativos pueden llegar a ser analizados por los usuarios, permite entender el proceso por el cual un contenido y un usuario interactúan para alcanzar diferentes niveles de convencimiento y, por ende, acciones diferentes.

Figura 2. Modelo de probabilidad de elaboración ELM, en base a revisión bibliográfica.

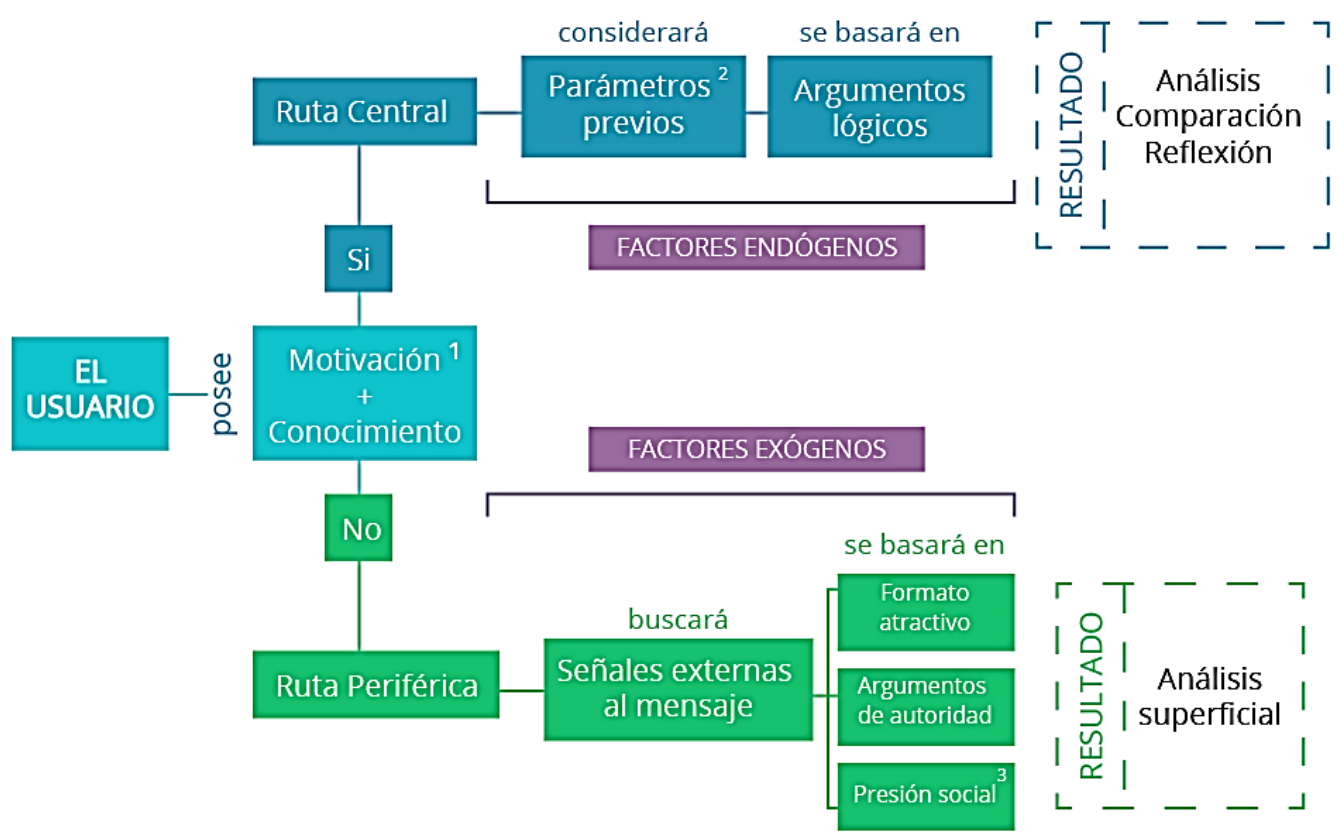

Fuente: Las Autoras (2019).

1. El usuario posee la motivación y el conocimiento necesario para elaborar sobre el tema expuesto; 2 . Considerará información adquirida en el pasado para la relacionarla con el tema expuesto; 3 . Surgen fenómenos como el FoMo y el SMF.

Con lo descrito previamente, el modelo conceptual de investigación, como se demuestra en la figura 3, quedó establecido de la siguiente manera: 
Figura 3. Modelo de investigación.

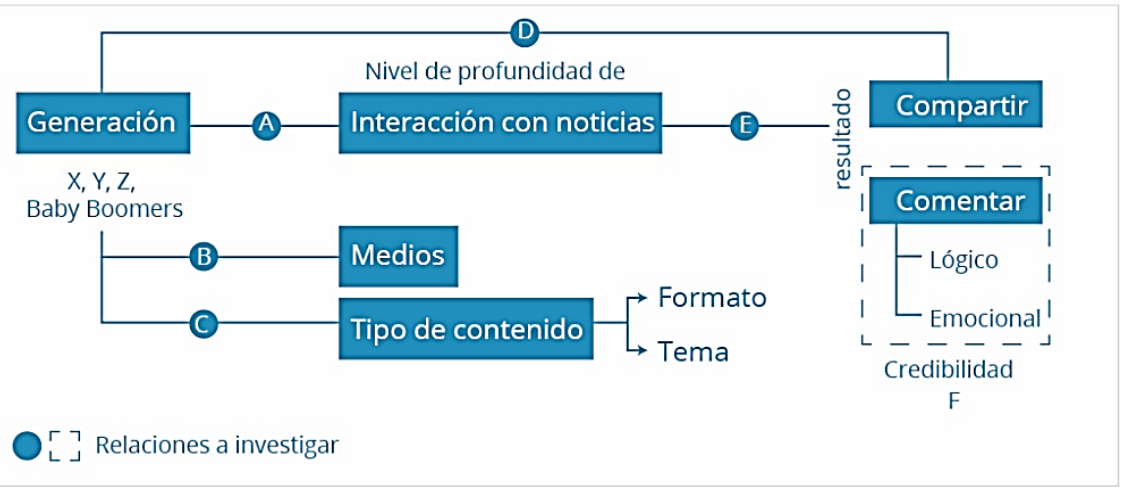

Fuente: Las Autoras (2019).

En base a este modelo, la hipótesis del estudio supone que las diferentes generaciones tienen diferentes comportamientos que influyen en su interacción con las noticias falsas.

\subsection{Relaciones por investigar}

La figura 3, resume las diferentes relaciones a investigar en el estudio. Se parte de la generación (edad) como variable independiente y se toman los medios utilizados, tipo de contenidos preferidos, nivel de profundidad de interacción con noticias y la probabilidad de compartir como variables dependientes. Sin embargo, el nivel de profundidad de interacción con noticias se convierte en variable independiente para efectos de análisis de la probabilidad de compartir. En las variables relacionadas con la acción de comentar no existía una dependencia clara entre ellas al momento de la construcción del modelo conceptual. Con estas consideraciones, las relaciones a investigar entre las variables presentadas fueron las siguientes:

a). Generaciones y nivel de profundidad de interacción con noticias: Se establecieron cinco niveles diferentes de profundidad de interacción con noticias en redes sociales, expresado en la tabla 2 , en base a 
comportamientos que los usuarios pueden tener al momento de procesar contenido informativo. Con ello la primera pregunta de investigación es: P1. ¿Las diferentes generaciones alcanzan diferentes niveles de profundidad de interacción con noticias?

Tabla 2. Niveles de profundidad de interacción con noticias.

Nivel 1 No veo noticias en redes sociales.

Nivel 2 Solo veo el titular y la descripción.

Nivel 3 Abro la noticia y la reviso rápidamente.

Nivel 4 Abro la noticia y la leo completa.

Nivel 5 Abro la noticia, la leo y busco fuentes o información complementaria.

Fuente: Las Autoras (2019).

b). Generaciones y medios preferidos: Se analizó la relación entre edades, redes sociales favoritas y medios informativos preferidos. Por ello la segunda pregunta para el estudio es: $\mathbf{P 2}$. ¿La preferencia por redes sociales y medios de información determinados está marcada por la edad?.

c). Generaciones y tipos de contenido: Se analizó la relación entre edades formatos favoritos, contenidos compartidos y contenidos preferidos en base a temáticas. La tercera y cuarta pregunta son: $\boldsymbol{P}$ 3. ¿Las generaciones mayores prefieren contenidos en formatos más tradicionales?, P4. ¿Las generaciones mayores prefieren contenidos de temáticas controversiales mientras que las generaciones más jóvenes buscan contenido personal que les aporte como individuos?.

d). Generaciones y fake news compartidas: Se analizó en qué generaciones se presentaron más noticias falsas compartidas durante un evento específico. La quinta pregunta es: P5. ¿Las generaciones mayores comparten más noticias falsas?.

e). Nivel de profundidad de interacción con noticias y fake news compartidas: Se analizó como los diferentes niveles de interacción con noticias, como se muestra en la tabla 2, conducían a compartir o no fake news. 
La sexta pregunta es: P6. ¿Los usuarios que tienden a leer las noticias completas tienen menos probabilidad de compartir noticias falsas?

f). Credibilidad obtenida de noticias falsas y carácter de reacciones a las mismas: Se analizó como casos particulares de noticias falsas alcanzaron diferentes niveles de credibilidad y si el cuestionamiento a una fuente implica un análisis impulsado por argumentos lógicos o emocionales. En tal sentido, la séptima pregunta es: P7. ¿Los usuarios que se guían por argumentos lógicos tienen mayor probabilidad de identificar noticias falsas que aquellos que reaccionan a la información basados en emociones personales?

\subsection{Métodos de recolección de datos}

\subsubsection{Encuestas}

Se realizó una encuesta con 9 preguntas que consideran los elementos planteados en el modelo conceptual de investigación. El muestreo utilizado fue el aleatorio simple. La población utilizada fue habitantes del Ecuador entre 17 y 74 años que tengan acceso a un teléfono móvil inteligente. Según datos proyectados del Instituto Nacional de Estadística y Censos (INEC, 2012): para el año 2019, la población en este rango de edad es de 11.114.201 de habitantes. Según datos proyectados del Instituto Nacional de Estadística y Censos (INEC, 2018): de estos el $41.4 \%$ cuenta con un teléfono inteligente. La población total entonces es de 4.601.279 de habitantes.

Considerando un error del $5 \%$ con un nivel de confiabilidad del $95 \%$, la muestra a encuestar fue de 385 personas. Cada rango etario explicado en la tabla tuvo una representación de entre el $17 \%$ y el $34 \%$ de la muestra total. Las encuestas fueron aplicadas por medio de WhatsApp de manera anónima durante la última semana de octubre y el mes de noviembre del año 2019. Este rango temporal coincidió con la coyuntura del paro nacional en Ecuador, lo cual sirvió de contexto para la investigación. Los datos recolectados en las encuestas y su análisis responden a las relaciones A, B, C, D y E del modelo 
conceptual, como se visualizó en la figura 3.

\subsubsection{Análisis de casos}

Se detectaron noticias falsas que circularon por Facebook en la primera semana de octubre, al inicio del paro nacional del año 2019 en Ecuador que se dio como respuesta a medidas económicas planteadas por el gobierno. Dos noticias falsas en este contexto fueron seleccionadas para la investigación por similitudes que los protagonistas de las noticias tienen en cuanto a argumentos de autoridad. La primera noticia hace referencia a Jamie Nebot y la segunda a Rafael Correa.

El análisis consideró los primeros 200 comentarios registrados en los posts originales y tras descartar aquellos comentarios no óptimos para análisis se tomaron en cuenta 189 y 190 comentarios en las noticias de Nebot y Correa respectivamente. Cada comentario, fue calificado como se expresa en la tabla 3.

Tabla 3. Criterios de calificación para comentarios.

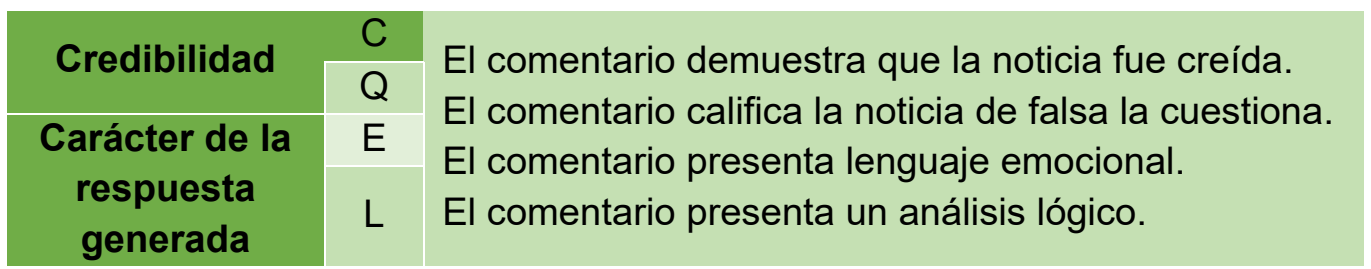

Fuente: Las Autoras (2019).

Este análisis responde a la relación $\mathrm{F}$ del modelo conceptual, que se expresó en la figura 3.

\section{Resultados}

3.1. Relación A: Generaciones y nivel de profundidad de interacción con noticias

P1: ¿Las diferentes generaciones alcanzan diferentes niveles de 
profundidad de interacción con noticias?. Los resultados de esta relación se pueden observar en el gráfico 1. La generación que más practica el leer una noticia completa es la X donde un $20 \%$ de encuestados pertenecientes a este rango etario asegura estar en el nivel 5 . La generación que menos practica el ver noticias en redes es la de los Baby Boomers donde un $15 \%$ de encuestados pertenecientes a este grupo, afirmó estar en el nivel 1. El nivel más común de interacción consiste en la apertura y revisión rápida de la noticia, recogiendo a más del $25 \%$ de usuarios dentro de cada generación. Esto responde a la $\mathbf{P 1}$, de manera afirmativa, pero no permite establecer una relación clara entre edad y profundidad de interacción con noticias ya que dentro de la misma generación hay muchos comportamientos que alcanzan porcentajes mayores al $20 \%$.

Gráfico 1. Nivel de profundidad de interacción con noticias según generación.

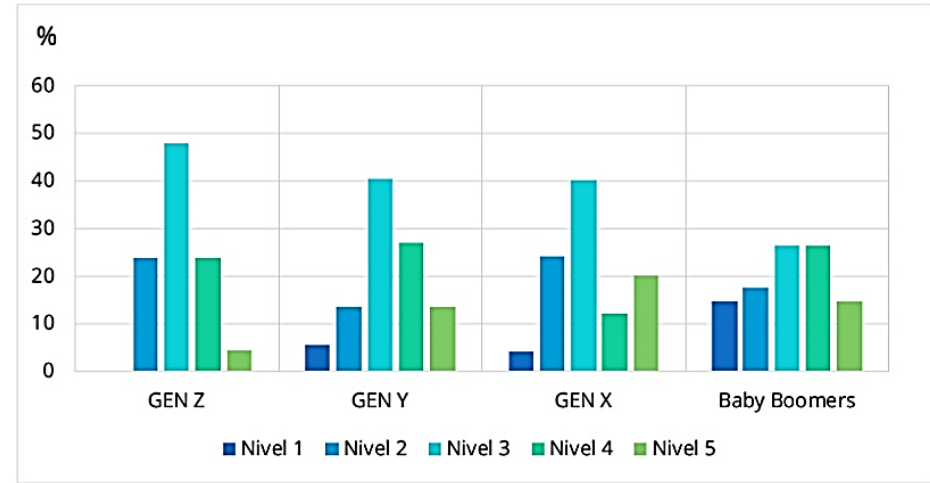

Fuente: Las Autoras (2019).

\subsection{Relación B: Generaciones y medios preferidos}

P2: ¿La preferencia por redes sociales y medios de información determinados está marcada por la edad?. La encuesta permitió responder a esta pregunta de manera afirmativa y arrojó resultados interesantes sobre cada generación y sus preferencias de redes sociales: En la Gen Z el 80\% prefiere Instagram y solo el 7\% prefiere Facebook. En la Gen Y el 52\% prefiere 
Instagram y el 38\% prefiere Facebook. En la Gen X el 64\% prefiere Facebook y solo el $20 \%$ prefiere Instagram. Por último, los Baby Boomers el $35 \%$ prefiere Facebook, el $26 \%$ Pinterest y el 18\% Instagram. Es la única generación donde Twitter supera el $10 \%$, con un $12 \%$ de preferencia y la única donde Linkedln aparece con $6 \%$ de preferencia.

Así mismo, en cuanto a medios informativos los resultados muestran tendencias por edad. Las redes sociales son el medio informativo favorito de la Gen Z, Y, y Baby Boomers. La preferencia por la televisión alcanza el mismo nivel que la preferencia por las redes sociales en la Gen X. Por otro lado, tanto la prensa impresa como digital, alcanzan menos del $10 \%$ de preferencia en todas las generaciones. Finalmente, la radio alcanza un $18 \%$ de preferencia en la generación de Baby Boomers.

\subsection{Relación C: Generaciones y tipos de contenido}

P3: ¿Las generaciones mayores prefieren contenidos en formatos más tradicionales?. El estudio responde a esta pregunta de manera inconcluyente, ya que todas las generaciones el formato preferido son imágenes con textos explicativos antes que videos o fotografías solas. La edad no influye de manera importante en la preferencia de formatos determinados.

P4: ¿Las generaciones mayores prefieren contenidos de temáticas controversiales mientras que las generaciones más jóvenes buscan contenido personal que les aporte como individuos?. En la encuesta se propusieron varios titulares de carácter polémico y otros neutrales. La generación que más preferencia mostró por titulares de carácter neutral como "Consejos de productividad para aumentar tu desarrollo profesional" fue la Z. Mientras que la preferencia por el titular "La falló a Correa el golpe de estado" crecía a medida que aumentaba la edad (Gen Z 20\%, Gen Y $41 \%$, Gen X $36 \%$ y Baby Boomers $50 \%$ ) La respuesta a la $\mathbf{P} \mathbf{4}$, es afirmativa puesto que a mayor edad aumenta la preferencia por titulares controversiales. 


\subsection{Relación D: Generaciones y noticias falsas compartidas}

P5: ¿Las generaciones mayores comparten más noticias falsas?. Se puede observar en el gráfico 2, que existe una relación directamente proporcional entre edad y probabilidad de compartir noticias falsas siendo los Baby Boomers quienes más comparten noticias falsas y la generación Z, los que menos lo hacen.

Gráfico 2. Relación con noticias falsas por edad.

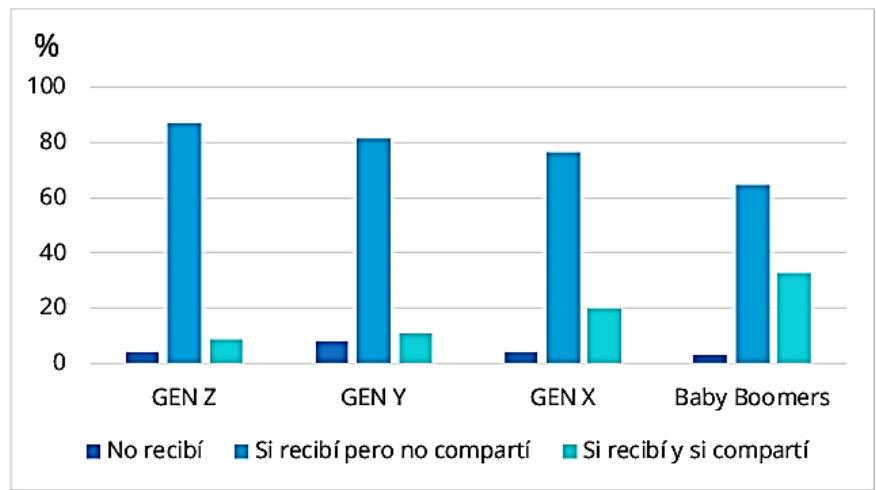

Fuente: Las Autoras (2019).

\subsection{Relación E: Nivel de profundidad de interacción con noticias y noticias falsas compartidas}

P6: ¿Los usuarios que tienden a leer las noticias completas tienen menos probabilidad de compartir noticias falsas?. La encuesta arrojó datos que responden de manera inconcluyente a esta pregunta cómo se puede ver en el gráfico 3. Las personas que compartieron noticias falsas son las que menos tienden a leer las noticias completas y verificarlas (nivel 5), siendo solo el $8 \%$ de personas que compartieron una noticia falsa las que afirman estar en el nivel 5. Sin embargo, el mismo grupo de personas que compartió una noticia falsa es el que más afirma estar en el nivel 4 de interacción, abrir la noticia y leerla completa, siendo un $29 \%$ el que afirma estar en este nivel. 
Gráfico 3. Noticias compartidas en relación a nivel de profundidad de interacción.

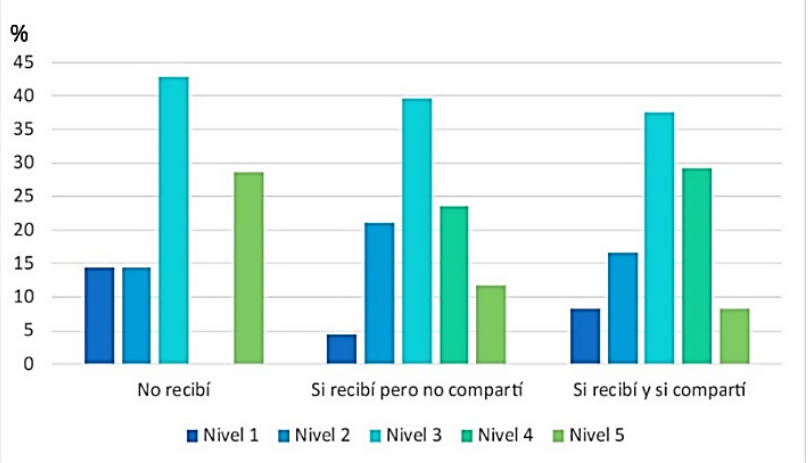

Fuente: Las Autoras (2019). 
Instituto Internacional de Investigación y Desarrollo Tecnológico Educativo INDTEC, C.A.

DOI: https://doi.org/10.29394/Scientific.issn.2542-2987.2020.5.16.11.208-229

OAI-PMH: http://www.indteca.com/ojs/index.php/Revista_Scientific/oai

Artículo Original / Original Article

Estos resultados responden de manera inconcluyente a al $\boldsymbol{P 7}$.

\section{Conclusiones}

En base al modelo conceptual planteado se puede concluir que existen varias relaciones entre contenidos, usuarios y comportamientos que son importantes en el proceso informativo. Estas relaciones, al ser analizadas en conjunto con rangos etarios de usuarios de redes sociales, indican que existen ciertos comportamientos propios de cada generación.

Como explica la teoría del ELM cada individuo está motivado de diversas maneras y tiene conocimientos en diferentes temas. Estas motivaciones posiblemente no tienen relaciones estrechas con edades, sino con experiencias personales que configuran la capacidad analítica de cada persona. Por ello, un estudio futuro que considere como base gusto personales o profesión podría arrojar resultados complementarios. Cabe resaltar que el modelo conceptual teórico creado para esta investigación, puede utilizarse reemplazando la variable de edad por alguna de las mencionadas.

En cuanto a la relación del estudio con la teoría revisada, se puede decir que el contexto político en el que se realizó la investigación demuestra el uso que se da a las noticias falsas para persuadir a las personas. Es importante destacar que el $95 \%$ de encuestados recibió noticias falsas durante el paro, lo cual confirmaría que el entorno digital ecuatoriano es muy parecido al planteado en la teoría y busca fines parecidos.

El presente estudio, logró cumplir su objetivo de analizar comportamientos y tipos de contenidos presentes en el mundo de las noticias falsas en Ecuador. Sin embargo, el realizar un muestreo más grande y considerar más casos de noticias falsas, podría ayudar a esclarecer dudas y reafirman los hallazgos. En cuanto a la hipótesis general, efectivamente se evidencian diferentes comportamientos en las diferentes generaciones, pero el porqué de estos, aún no está claro. 


\section{Referencias}

Allcott, H., Gentzkow, M., \& Yu, C. (2019). Trends in the diffusion of misinformation on social media. Research \& Politics, 6(2), 1-8, ISSN: 2053-1680; e-ISSN: 2053-1680. Recovered from: https://doi.org/10.1177/2053168019848554

Bakir, V., \& McStay, A. (2017). Fake News and The Economy of Emotions. Digital Journalism, 6(2), 154-175, e-ISSN: 2167-0811. Recovered from: https://doi.org/10.1080/21670811.2017.1345645

Calderón, F. (2019). Impacto de las nuevas tecnologías en la masificación de la educación. Revista Scientific, 4(Ed. Esp.), 173-187, e-ISSN: 2542-2987. Recuperado de:

https://doi.org/10.29394/Scientific.issn.2542-2987.2019.4.E.10.173$\underline{187}$

Costa, J. (2012). EI DirCom hoy: dirección y gestión de la comunicación en la nueva economía. 3ra. edición, Serie nro. 4, ISBN: 978-84-6126816-0. Barcelona, España: Editorial CPC.

Elangovan, N., \& Rajendran R. (2015a,b). Conceptual Model: A Framework for Institutionalizing the Vigor in Business Research. Conference, ISBN: 9-38423-409-X. India: Sri Ramakrishna Institute of Technology.

Gelfert, A. (2018). Fake News: A Definition. Informal Logic, 38(1), 84-117, eISSN: 0824-2577. Recovered from:

https://doi.org/10.22329/il.v38i1.5068

INEC (2012). Proyecciones Poblacionales. Ecuador: Instituto Nacional de Estadística y Censos. Recuperado de:

https://www.ecuadorencifras.gob.ec/proyecciones-poblacionales/

INEC (2018). Tecnologías de la Información y Comunicación-TIC. Ecuador:

Instituto Nacional de Estadística y Censos. Recuperado de:

https://www.ecuadorencifras.gob.ec/tecnologias-de-la-informacion-ycomunicacion-tic/ 
Instituto Internacional de Investigación y Desarrollo Tecnológico Educativo INDTEC, C.A.

DOI: https://doi.org/10.29394/Scientific.issn.2542-2987.2020.5.16.11.208-229

OAI-PMH: http://www.indteca.com/ojs/index.php/Revista_Scientific/oai

Artículo Original / Original Article

Kotler, P., \& Keller, K. (2012). Dirección de Marketing. Decimocuarta edición, ISBN: 978-607-32-1245-8. México: Editorial Pearson Educación.

Maharjan, P. (2018). Elaboration Likelihood Model. Businesstopia. Bagmati, Nepal: Parewa Labs Pvt. Ltd. Recovered from:

https://www.businesstopia.net/communication/elaboration-likelihood$\underline{\text { model }}$

Mustafaraj, E., \& Metaxas, P. (2017a,b). The Fake News Spreading Plague:

Was it Preventable?. ArXiv.org, 1-6, e-ISSN: 2331-8422. Recovered

from: https://arxiv.org/abs/1703.06988

Nielsen (2015). Estilos de Vida Generacionales: Cómo vivimos, comemos, jugamos, trabajamos y ahorramos para nuestro futuro. EE.UU.: The

Nielsen Company, LLC. Recuperado de: https://www.nielsen.com/wpcontent/uploads/sites/3/2019/04/EstilosdeVidaGeneracionales.pdf

Petty, R., \& Cacioppo, J. (1986). The Elaboration Likelihood Model of Persuasion. Advances in Experimental Social Psychology, (19),123205, e-ISSN: 0065-2601. Recovered from:

https://doi.org/10.1016/s0065-2601(08)60214-2

Ravindran, T., Yeow, A., \& Hoe, D. (2014). Antecedents and effects of social network fatigue. Jasist: Journal of the Association for Information Science and Technology, 65(11), 2306-2320, e-ISSN: 2330-1643. Recovered from: https://doi.org/10.1002/asi.23122

Talwar, S., Dhir, A., Kaur, P., Zafar, N., \& Alrasheedy, M. (2019). Why do people share fake news? Associations between the dark side of social media use and fake news sharing behavior. Journal of Retailing and Consumer Services, 51, 72-82, e-ISSN: 0969-6989. Recovered from: https://doi.org/10.1016/j.jretconser.2019.05.026

Vives, S. (2019a,b). Posverdad. La nueva guerra contra la verdad y cómo combatirla. Clivatge, (7), 286-297, e-ISSN: 2014-6590. Recuperado de: http://revistes.ub.edu/index.php/clivatge/article/view/28602/29193 
OAI-PMH: http://www.indteca.com/ojs/index.php/Revista_Scientific/oai

Artículo Original / Original Article

\section{Michelle Alexandra Benítez Vite}

e-mail: mia77bv@gmail.com

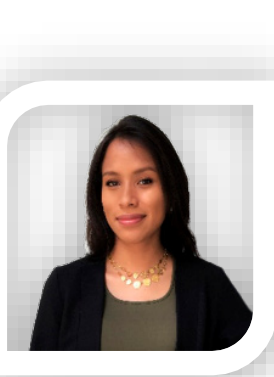

Nacida en Quito, Ecuador, el 17 de julio del año 1997. Licenciada en Comunicación Organizacional y Relaciones Públicas por la Universidad Internacional del Ecuador (UIDE); Poseo experiencia en creación e implementación de campañas y planes de comunicación interna y externa; Dentro de mi trayectoria, se encuentra la participación como asistente de dirección de extras, en la película ecuatoriana Agujero Negro y asistente de producción en la película Sansón; En el campo de la publicidad, me destaco como finalista de los concursos Effie College Ecuador (2016) y Effie College Ecuador (2017); Soy Ganadora del primer lugar del concurso nacional de publicidad INK HOUSE (2018) de Mullen Lowe Delta. 


\section{Artículo Original / Original Article}

\section{Patricia Elizabeth Hidalgo Albuja \\ e-mail: phidalgo@uide.edu.ec}

Nacida en Quito, Ecuador, el 9 de abril del año 1960.

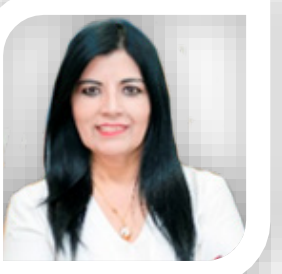

Doctora en Ciencias Sociales por la Facultad Latinoamericana de Ciencias Sociales (FLACSO), Ecuador; Directora de la Escuela de Comunicación de la Universidad Internacional del Ecuador (UIDE); Mi trabajo de investigación y docencia, con 25 años de experiencia, me centro sobre cinco campos específicos que son: la comunicación política, la comunicación organizacional, la planificación estratégica, la democracia participativa y la gestión local; Soy autora del libro Claros y Oscuros de la Democracia Participativa: El caso de las asambleas de presupuesto participativo del Distrito Metropolitano de Quito 2010-2013, publicado en el año 2018; Participo como miembro activo de la Asociación Mexicana de Ciencias Políticas (AMECIP), Asociación Latinoamericana de Ciencia Política (ALACIP), y la Red de Politólogas y del Consejo de Investigación de la Universidad Internacional del Ecuador (UIDE).

El contenido de este manuscrito se difunde bajo una Licencia de Creative Commons ReconocimientoNoComercial-Compartirlgual 4.0 Internacional 